10-1-2016

\title{
Effects of Olfactory Sense on Chocolate Craving
}

Michael W. Firmin

Aubrey L. Gillette

Taylor E. Hobbs

Di Wu

Follow this and additional works at: https://digitalcommons.cedarville.edu/psychology_publications

Part of the Psychology Commons

This Article is brought to you for free and open access by

DigitalCommons@Cedarville, a service of the Centennial

Library. It has been accepted for inclusion in Psychology

Faculty Publications by an authorized administrator of

DigitalCommons@Cedarville. For more information,

please contact digitalcommons@cedarville.edu. 


\title{
Effects of olfactory sense on chocolate craving
}

\author{
Michael W. Firmin*, Aubrey L. Gillette, Taylor E. Hobbs, Di Wu \\ Cedarville University, 251N. Main Street, Cedarville, $\mathrm{OH}, 45314$, United States
}

\section{A R T I C L E I N F O}

\section{Article history:}

Received 15 May 2016

Received in revised form

28 June 2016

Accepted 4 July 2016

Available online 6 July 2016

\section{Keywords:}

Craving

Chocolate

Weight loss

Essential oils

Olfactory

\begin{abstract}
A B S T R A C T
In the present study, we assessed the effect of the olfactory sense on chocolate craving in college females. Building on previous research by Kemps and Tiggemann (2013), we hypothesized that a fresh scent would decrease one's craving level for chocolate food. While the precursor study only addressed the decrease of chocolate craving, we also hypothesized that a sweet scent would increase one's craving level for chocolate foods. In the present experiment, participants rated their craving levels after viewing images of chocolate foods and inhaling essential oils: one fresh (Slique ${ }^{\mathrm{TM}}$ essence), and one sweet (vanilla). Results supported both of the hypotheses: inhaling a fresh scent reduced females' craving levels; similarly, when a sweet scent was inhaled, the participants' craving levels for chocolate food increased. These findings are particularly beneficial for women seeking weight loss and the findings can be applied in contexts such as weight loss programs, therapy, and maintenance programs, even beyond college settings. The results are particularly useful for helping women regarding stimuli that might serve as triggers for chocolate cravings.
\end{abstract}

() 2016 Elsevier Ltd. All rights reserved.
Individuals frequently say that they "crave" chocolate, or they "crave" carbohydrates but this subjective term is not typically defined very operationally. Craving is a function of one's senses and neural networking, and is more complicated than one might predict. Rozin (1982) notes that people's taste is influenced by their sense of smell, otherwise known as olfaction. The olfactory sense is one of the key factors in desiring, or craving, food (Tiggemann \& Kemps, 2005).

Women most often crave sweet, carbohydrate and fat-rich foods; sugar itself is increasingly being considered an addictive agent (Avena, Rada, \& Hoebel, 2008; Christensen, 2007). Christensen reported that people who are prone to craving-otherwise known as cravers-have higher energy intakes than they need, and the majority of their intake is carbohydrates, of which sugars are the most represented. Christensen's review also determined that cravers' body mass index (BMI) tends to be higher than that of noncravers. Furthermore, individuals with a higher BMI tend to fantasize more frequently about the foods they crave (Delahanty, Meigs, Hayden, Williamson, \& Nathan, 2002). A study by Stafford and Whittle (2015) identified an association between obese individuals (having a BMI greater than or equal to 30) and a greater sensitivity to chocolate odor compared to a non-obese group. These

\footnotetext{
* Corresponding author.

E-mail address: firmin@cedarville.edu (M.W. Firmin).
}

findings give rise to the question of sugar potentially being an addictive substance. Since sugar appears to be a culprit for cravers developing poor overeating habits, there is a possibility of the substance having addictive attributes.

In the realm of addiction, Avena et al. (2008) reported that craving is a fundamental aspect of dependence, following the stages of bingeing and withdrawal. These authors reviewed the concept of sugar dependence in an animal model, finding neurochemical changes within the brain that are also identified as a result of addictive drug use. These changes include dopamine adaptations, opioid receptor binding, and other reactions in the nucleus accumbens, a part of the brain heavily involved in the experience of pleasure and addiction. While craving does not only occur in addicts, it nevertheless has major implications for weight management, particularly in those who are genetically predisposed toward obesity (Christensen, 2007; Davis \& Carter, 2014).

The word addiction first appeared in a health journal in 1890, referring to chocolate (Davis \& Carter, 2014). Chocolate persistently appears to be one of America's most craved foods (Jáuregui-Lobera, Bolaños-Rios, Valero, \& Prieto, 2012). Rolls and McCabe (2007) utilized fMRI to measure 16 females' responses to the flavor and sight of chocolate. Eight of these females were identified as cravers, and the other eight were non-cravers, based on a chocolate cravers questionnaire, developed by Cepeda-Benito, Fernandez, and Moreno (2003). The fMRI data revealed differences in cravers' and non-cravers' responses to sensory components of chocolate, 
which was a highly craved food, as indicated by "blood oxygenation level-dependent signals in the pregenual cingulate cortex and medial orbitofrontal cortex" (Rolls \& McCabe, 2007, p. 1067). Furthermore, the results were influenced by chocolate's subjective pleasantness, suggesting that chocolate is a hedonic, desirable, and palatable food worth studying further.

People's mental image of food significantly affects their experience of craving. Mental imagery has been used in order to determine the level of craving for particular foods, revealing chocolate as a most-craved food (Jáuregui-Lobera et al., 2012). Researchers assessed undergraduate students' imaging ability using Bett's Questionnaire Upon Mental Imagery, and had the students imagine that they were eating their favorite food. Sweet foods were among the most intensely craved foods, and intensity was correlated with phrases such as "I imagined the smell/taste of it" and "I pictured myself having it" (Jáuregui-Lobera et al., 2012, p. 1931). The study was founded upon two different theories of craving: Tiffany's theory and Elaborated Intrusion theory. The Elaborated Intrusion Theory of desire suggests "emotive imagery and associated sensations are especially important in craving because somatosensory links contribute a particular piquancy and motivational power to the experience" (Kavanagh, Andrade, \& May 2005, p. 446). During elaboration, individuals may ruminate on the food or substance of desire and concentrate on the pleasurable aspects of the substance instead of whatever task they were focused on before the intrusion. This particular theory has stark implications for craving: it potentially can take over one's cognitive faculties through mental imagery and, therefore, mental imagery may be a necessary tool in order to decrease those same cravings. Dieting can ironically increase one's craving for food, much like thought suppression has a counterintuitive effect on one's thoughts (Hill, 2007). However, fasting potentially can successfully decrease food cravings. The presence of stimuli, repetition of thoughts, and mental imagery all play a role in one's level of craving.

Mental imagery may also be useful in treatment regarding food cravings. Kemps and Tiggemann (2015) suggested utilizing mental imagery techniques within cognitive-behavioral therapy for the purpose of craving reduction. Additional suggested self-help tools include olfactory and visual exercises. Moffitt, Brinkworth, Noakes, and Mohr (2012) found that cognitive diffusion leads to more improvement in eating behavior than does cognitive restructuring. These techniques are models of promoting health and weight loss among obese individuals but also can be used in the general population. It is possible that olfactory sense, paired with cognitive diffusion, would have an even greater impact on healthy eating efforts. Essential oils such as peppermint are currently being studied for their efficacy in medical treatment (Haber \& El-lbiary, 2016). Peppermint is helpful in the management of Irritable Bowel Syndrome and other pain and discomfort-related medical problems. Likewise, numerous other essential oils may have medically therapeutic effects (Edris, 2007).

In a precursor study to the present one, Kemps and Tiggemann (2013) assessed the effect of olfactory sense on craving by presenting images of both sweet and savory food and having participants smell a neutral scent and listen to an auditory recording. This within-subjects design included 56 female undergraduate students. Researchers chose to include only female participants due to prior research by Weingarton and Elston (1991) that demonstrated a higher prevalence of food cravings among women, as compared to men. Participants were allowed to view each image for $5 \mathrm{~s}$, then smell the neutral scent (methyl acetate), listen to the recording, or do nothing during an $8 \mathrm{~s}$ retention period. The images were presented in a counterbalanced order, with participants assessed individually. Kemps and Tiggemann found that the smell of methyl acetate decreased the participants' craving for sweet foods more than the auditory and control conditions. This finding supports the concepts food cravings and the conflict between cravings' olfactory nature and odors for mental resources. These researchers also conducted the study using chocolate foods instead of the mixed sweet and savory foods, finding similar results. With 57 female participants who were separate from their first study, researchers found that reported chocolate cravings were significantly lower in the olfactory condition than they were in the auditory and control conditions. This finding led the present researchers to explore the olfactory sense's role in craving, as influenced by sweet and fresh smells. Instead of presenting an auditory condition, we chose to test both a sweet and a fresh smell, and created an olfactory-only design. The reason for selecting this protocol is because the auditory condition was not as successful in Kemps and Tiggemann's study as was the olfactory condition.

In the present study, we sought to determine what effect the olfactory sense may have on craving, specifically craving for chocolate, which is a reportedly craved and pleasurable food. Through replication of Kemps \& Tiggemann, 2013 study, which also assessed women's chocolate cravings, we presented images of chocolate foods, followed by a retention period during which participants smelled the instructed scents before recording their level of craving for the food that was presented visually. While the previous study assessed the decrease in craving elicited by the neutral scent of methyl acetate, in the present study we sought to address the other end of the spectrum as well: the effect of a sweet scent on craving levels. We anticipated that a sweet scent would increase one's craving for chocolate food and a fresh scent would decrease one's craving for chocolate food, as is consistent with the findings of the initial study by Kemps and Tiggemann (2013). We utilized Young Living's Slique essential oil for the fresh condition and vanilla essential oil for the sweet condition. Previous studies have utilized essential oils to decrease food cravings. Reed, Almeida, Wershing, and Raudenbush (2008) used peppermint oil to reduce caloric intake. While peppermint is not an ingredient of Slique Essence, spearmint is, and these mints are arguably similar enough to produce a similar effect of curbing hunger and food intake. Additionally, Kemps, Tiggemann, and Bettany (2012) found that jasmine can curb chocolate craving. Jasmine oil comes from jasmine plants, which are flowering shrubs. Similarly, ocotea oil, a main ingredient in Slique Essence that helps with digestion, is the product of a flowering tree. Another study found that an orange scent decreased the intake of chocolate (Buckland, Finlayson, \& Hetherington, 2013). The citrus scent of oranges seemed to distract participants from their chocolate cravings. Spearmint, grapefruit, tangerine, lemon, and ocotea are ingredients of Slique Essence, thereby predicting decreased chocolate craving. We assumed in the present study that participants possessed a reasonable liking for chocolate food and that their olfactory sense would influence their desire for the chocolate food that was presented to them.

\section{Method}

\subsection{Participants}

Female undergraduate students were recruited through Physical Activity and General Psychology classes. Ninety-six female students participated in the study, while three responses were removed due to possessing insufficient data and one removed due to being underage, resulting in 92 participants in the final data set. These courses are required in the general education core of a Midwest, private, comprehensive university. Consequently, the sample represented various majors across the university and ages of participants ranged from 18 to 22 with the average being 19 . Congruent with the general overall demographic of the university where the 
study was conducted, $89 \%$ of the sample was Caucasian and $8 \%$ consisted of minority participants: $5 \%$ Hispanic, $2 \%$ Asian, and $1 \%$ Egyptian, while $3 \%$ of participants refrained from reporting their ethnicity. Twenty different states (and one foreign country) were represented by the various participants when identifying their residency. Consequently, the sample represented a relative crosssection of females spanning most of the United States (with the exception of African Americans, since they were not represented in this particular sample). Additionally, the mean of participants' selfreported body mass index (BMI) was 22.96. The range of participants' BMI was $16-43$, so while some students were underweight and others were obese, the average BMI constitutes a normal, healthy weight.

\section{Materials}

The stimuli were 12 digital colored photographs of chocolate foods in the categories of chocolate cake, chocolate muffin, chocolate ice cream, and chocolate brownie. There were three different images for each category of food, in order to have one image per condition. The 12 images were chosen through a pilot study of 16 female undergraduate students who selected three of nine images they thought best represented each category. The images were presented through PowerPoint software on a screen, sized $92^{\prime \prime} \times 164^{\prime \prime}$ using NEC NP-PA550W projectors with $1280 \times 800$ resolution.

Slique Essence essential oil, a Young Living product, was utilized as the fresh scent. This product is described as a "citramint" scent, combining citrus and mint scents to curb appetite between or during meals (Young Living). Slique Essence's intended use is to enhance beverages, although it may also be inhaled, as it was during the present study. Vanilla essential oil, manufactured by Fabulous Frannie, was utilized as the sweet scent. Since it is a standard scent and not a unique blend, we chose not to use Young Living vanilla oil. Participants did not rate their perception of these two odors, and proceeded through the experiment despite their variety of perceptions. These two essential oils were presented in petri dishes (size $100 \mathrm{~mm} \times 15 \mathrm{~mm}$ ), which were labeled using markers that simply indicated " 1 " and " 2 ," so as not to reveal the contents of each dish. Approximately two drops of essential oil (equivalent to about $1 / 10 \mathrm{ml}$ ) were placed into each dish, in order to be inhaled directly at the time of instruction. A $100 \mathrm{~mm}$ visual analogue scale was used for participants to rate their craving levels for each image.

\subsection{Procedure}

Participants were tested in groups ranging from 3 to 33 individuals at a time. They sat (spaced-out) in the front-center sections of a lecture room facing a projector screen. Male students were asked to leave the room before the informed consent and description of the present study were read to the women. As such, the males did not possess knowledge regarding the details of this study, nor were they present during any of the photo and oil inhalation trials. After agreeing to participate, the females first completed two practice trials in order to understand the way the images would be presented and to practice recording their level of craving on the $100 \mathrm{~mm}$ visual analogue scale. The participants then completed 12 trials, with four images (one per category) presented under each condition (fresh scent, sweet scent, and control). The condition orders were counterbalanced by group so that each image was shown in all three conditions (control, sweet, and fresh) throughout the study. All 12 photos were presented in the same order for all three groups, but the stimulus condition changed according to the group assignment. Each group participated in the experiment in separate time sessions so that instructions regarding which scent to inhale were clearly understood by the participants.

During the 8-s retention period after each image, participants were instructed to smell oil 1 (vanilla), oil 2 (Slique Essence), or not smell anything. These two essential oils were presented in individual petri dishes which were placed at each participant's table in the lecture room, next to their informed consent forms and rating response packets. After they smelled the indicated scent, participants recorded their level of craving on a $100 \mathrm{~mm}$ visual analogue scale. The procedure took less than $10 \mathrm{~min}$ for the participants to complete in the small group setting. Response forms were collected for analysis (see Table 1 ).

\section{Results}

The craving ratings were averaged across the four trials of chocolate foods in each olfactory scent condition. Descriptive statistics for the overall craving ratings and the three order groups can be seen in Table 2 . We conducted a $3 \times 3$ mixed-design ANOVA with scent condition (control, sweet scent, fresh scent) as a withinsubjects factor and group (Group1, 2, 3) as a between-subjects factor. There was a significant scent condition $\times$ group interaction $(F(3.34,128.71)=3.18, p<0.05)$. Therefore, further analyses for the three groups were examined separately for the effect of olfactory simulations.

The one-way repeated measures ANOVA for Group 1 yielded a significant main effect of the scent condition, $F$ (1.30, $29.82)=24.67, p<0.001$. As predicted, the results of post-hoc multiple comparisons using a Bonferroni correction showed that the craving ratings were significantly higher in the sweet than in the fresh condition, $t(23)=5.31, p<0.001$, and in the control condition $t(23)=3.54, p<0.01$. Moreover, the ratings in the fresh condition were significantly lower than those in the control condition $t(23)=4.88, p<0.001$. The same significant main effect of olfactory scent condition was found in Group $3, F(2,60)=29.63$, $p<0.001$. Similarly, craving ratings were higher in the sweet than those in the fresh condition $t(30)=6.81, p<0.001$, and in the control condition $t(30)=3.37, p<0.01$. The significant rating difference was also found between the fresh and control condition, $t(30)=4.66, p<0.001$.

Although the same significant effect of the scent condition was presented in Group 2, $F(1.83,44.02)=23.87, p<0.001$, the results of multiple comparisons showed a different pattern. The craving ratings were still significantly higher in the sweet condition than

Table 1

Group order designations: photos 1, 5, and 9 are of cake; photos 2, 6, and 10 are of muffins; photos 3,7 , and 11 are of ice cream; photos 4,8 , and 12 are brownies.

\begin{tabular}{llll}
\hline & Photos & & \\
\cline { 2 - 4 } & 1234 & 5678 & 9101112 \\
\hline Group 1 & Control & Sweet & Fresh \\
Group 2 & Fresh & Control & Sweet \\
Group 3 & Sweet & Fresh & Control \\
\hline
\end{tabular}

Table 2

Means and standard deviations for food craving ratings for the three scent conditions.

\begin{tabular}{|c|c|c|c|c|c|c|c|c|}
\hline & \multicolumn{2}{|l|}{ Total } & \multicolumn{2}{|c|}{ Group 1} & \multicolumn{2}{|c|}{ Group 2} & \multicolumn{2}{|c|}{ Group 3} \\
\hline & M & SD & M & SD & M & SD & M & SD \\
\hline Control & 46.24 & 20.42 & 50.97 & 19.05 & 41.29 & 18.54 & 46.58 & 22.48 \\
\hline Sweet & 59.24 & 20.21 & 60.30 & 23.45 & 62.34 & 17.39 & 55.92 & 19.79 \\
\hline Fresh & 33.82 & 21.78 & 32.54 & 23.60 & 39.53 & 18.90 & 30.20 & 22.24 \\
\hline
\end{tabular}


they were in the fresh scent $(t(24)=5.73, p<0.001)$ and control conditions $(t(24)=6.84, p<0.001)$. It was unexpected that the ratings in the fresh and control conditions did not differ significantly with each other $(t(24)=0.45, p>0.05)$. However, this should not lead to a quick conclusion that the fresh scent did not decrease the level of chocolate craving in Group 2.

Since the participants viewed two sets of four chocolate foods in the fresh scent and control conditions, it was possible that they had different levels of craving for the chocolate foods on these two sets of photos originally. That is, without any olfactory simulations, the craving ratings for the chocolate foods presented in the fresh scent condition of Group 2 should be higher and then lowered after the participants inhaled the fresh scent. The lowered craving ratings did not differ much from the ratings of the chocolate foods presented on the other set of photos in the control condition.

Therefore, it was reasonable to conduct additional one-way repeated measures ANOVA by using the photos of chocolate foods as cases in order to examine the main effect of scent condition. It allowed us to compare the different craving levels of the same chocolate foods between the three scent conditions. A significant main effect of the scent condition, $F(2,22)=72.63, p<0.001$, was found. The results of post-hoc multiple comparisons were consistent with our prediction. The craving ratings were significantly higher in the sweet $(M=59.66, S D=5.12)$ condition than they were in the fresh scent $(M=34.73, S D=4.90)$ condition, $t(11)=10.62$, $p<0.001$ and in the control $(M=47.03, S D=6.61)$ condition, $t(11)=5.56, p<0.001$. The ratings were significantly lower in the fresh scent condition than in the control condition, $t(11)=8.62$, $p<0.001$. The relationship between scent condition and craving level can be seen in Fig. 1.

The participants' self-reported body mass index (BMI) did not correlate with craving ratings, as previous research had suggested it might. Higher BMI levels did not correlate significantly with higher craving ratings, although this was not a major hypothesis of the present study. The mean BMI rating was 22.96, which is classified as a normal, healthy weight category.

\section{Discussion}

In the present study, we assessed the effect of olfactory sense on chocolate craving by way of subjects inhaling essential oils and recording craving levels. The results confirm the hypotheses that a fresh scent decreases one's craving-level for chocolate foods and a sweet scent increases one's craving level for chocolate foods. The finding that fresh scent decreased craving levels is consistent with that of the replicated study, where Kemps and Tiggemann (2013) found that a neutral scent decreased craving levels for chocolate foods. The second finding, that a sweet scent increased craving levels, adds to the findings of previous research. Both our present study as well as Kemps and Tiggemann's assume that chocolate is a

\section{Craving Averages According to Groups}

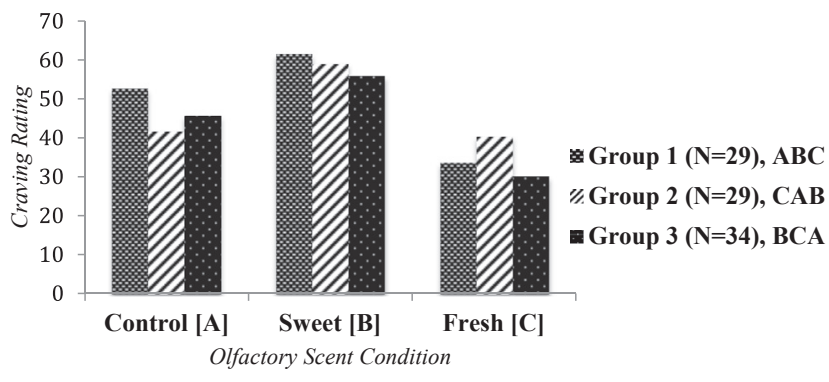

Fig. 1. Participant craving ratings, organized by group order. commonly craved food and that mental imagery powerfully influences one's craving levels (Jáuregui-Lobera et al., 2012). Furthermore, the present study demonstrates that one's olfactory sense plays an integral role in their level of craving. While previous research by Christensen (2007) and Delahanty et al. (2002) suggested a relationship between having a high BMI and both fitting the classification of "craver" and having higher levels of fantasy about their desired foods, the results of the present study did not confirm this claim. Those with low and high BMI reported similar craving ratings. The average BMI classification of "healthy" weight likely explains why there was no correlation between BMI level and craving ratings in the present study.

The findings of the present study possess numerous implications for health professionals, women seeking weight loss, both in and out of college settings, and the general public. Since the participants of this study are adults (18 and older), these findings should be relevant to older women as well, as they likely have similar cravings for chocolate. Health professionals may benefit from a newfound knowledge regarding the potential power of essential oils. Specifically, essential oil may be utilized as one factor in a treatment plan in order to reduce women's chocolate cravings. This protocol could be particularly beneficial in cases where women need to lose weight, but they also struggle with craving sweet foods. Slique Essence is sold as an essential oil that can be added to water (or other drinks) or inhaled, as occurred in this study. Additionally, the substance is sold in tea packets and other forms that potentially could be useful for women in weight loss programs. This essential oil, alongside mental imagery treatments, potentially could promote healthy life change (Kemps \& Tiggemann, 2015). As essential oils such as peppermint become increasingly popular in their therapeutic effects, more recently concocted oil mixtures may gain acceptance as well, but should be utilized carefully due to their potential to interact with other medicines (Haber \& El-lbiary, 2016). Primary care providers, nutritionists, psychologists, and the like may use mental imagery in order to assist their clients with craving issues, and they potentially could recommend an essential oil, or a similar product, as a more natural supplement than other weight loss helps. [As a disclosure, none of the present co-authors have any past or present financial or other type of affiliation with Slique ${ }^{\mathrm{TM}}$.]

Whether or not essential oils become utilized in psychotherapy, women can nonetheless choose to purchase this (or a similar product) in order to curb their appetite for sweet foods. Moreover, the findings of this study should help women identify the types of scents that trigger the influx and decline of their cravings for food. For example, sweet scents, such as vanilla, can exacerbate an already existing craving, so certain women desiring to curb weight gain may want to avoid such scents. In contrast, fresh scents such as the citramint scent of Slique Essence have the potential to decrease cravings and distract from one's urge to consume unhealthy foods, specifically chocolate foods. The finding that the smell and sight of oranges decreased female dieters' intake of chocolate because it is a diet-congruent food generates an insightful model for potentially decreasing chocolate cravings (Buckland et al., 2013). This model applies to the present study in that the fresh scent of slique the essential oil used in the present study is congruent with a healthy diet. One limitation of this mechanism is that we did not ask participants to rate whether or not they were dieting at the time of the experiment. If they were, then this phenomenon could presumably help explain the relationship between a fresh scent and a decreased desire for chocolate foods. Similarly, as previously mentioned, Kemps et al. (2012) found that jasmine is able to decrease chocolate cravings. This natural, flowering shrub is indicative of freshness and cleanliness and, therefore, constitutes a non-food distraction to food cravings. Reed et al. (2008) found peppermint to be effective in curbing food intake as well. Evidently, the important factor is not 
that the scent is a non-food aroma, since oranges, peppermint, and jasmine all have such reported similar effects. It seems that the most important mechanism for reducing chocolate craving is to inhale a scent that is opposite to the craved stimulus. For example, the smell of sugary doughnuts is much too similar to that of chocolate cake, so that scent is not a beneficial distraction-it could increase the craving level much as vanilla oil does. In contrast, fruits, vegetables, and flowers all have drastically different scents to that of vanilla and chocolate, and they appear to adequately redirect the neural pathways of the craving control center within the brain. Overall, an awareness of one's olfactory sense around sweet and fresh scents may be one key to fighting chocolate craving.

\subsection{Limitations and future research}

Limitations of the present study include utilizing a sample of college students who were mostly Caucasian. Future studies should consider obtaining non-college populations from diverse ethnic backgrounds and also replicating the present study with males. Additionally, as noted in the present study, we assumed that research participants would have a reasonable enjoyment of chocolate. Future researchers may wish to survey prospective participants and discover their self-reported levels of chocolate preference, controlling for this potential variable. The present study did not control for a variety of factors that may interact with sensory cues. While BMI was addressed as a possible influential factor, there other factors may also contribute to the outcomes of the present study (e.g., include hunger, anxiety, appetite level, and cognitive restraint, among others). We attempted to control for hunger, based on the timing of the experiments, although it was not easily controllable, since the General Psychology classes only met at 11 a.m. and 12 p.m. Nonetheless, we believe that most students in those courses did not eat lunch before the experiment, due to the stringency of class schedules on that particular Monday.

Beyond hunger and BMI, some outstanding factors may have had an interaction with the presented sensory cues, thereby allowing some space for random variation. Since these were not measured, we are unsure regarding their effect on the participants' craving ratings. Future researchers should consider the effects of other factors that may interact with sensory cues. Future researchers may also wish to expand the variety of stimuli presentations beyond Slique in order to enhance the study's external validity.

Multiple possibilities for future research exist regarding this topic. For example, a similar model could be replicated with a group of male participants in order to assess whether the essential oils have the same impact on their craving levels. In this case, a different set of images may need to be presented because men presumably do not tend to crave chocolate as strongly as women (Weingarten \& Elston, 1991). Additionally, future potential research can involve the assessment of essential oils and mental imagery efficacy in treatment programs. Participants could be gathered from a nutritionist or therapist and researchers may implement cognitive behavioral therapy paired with mental imagery and an essential oil prescription. This design could either fit in a between-subjects or within-subjects study. Several other future research potentials exist beyond these two, but these could be a sufficient starting point.

\section{References}

Avena, N. M., Rada, P., \& Hoebel, B. G. (2008). Evidence for sugar addiction Behavioral and neurochemical effects of intermittent, excessive sugar intake. Neuroscience and Behavioral Reviews, 32, 20-39.

Buckland, N. J., Finlayson, G., \& Hetherington, M. M. (2013). Pre-exposure to dietcongruent food reduces energy intake in restrained dieting women. Eating Behaviors, 14, 249-254.

Cepeda-Benito, A., Fernandez, M. C., \& Moreno, S. (2003). Relationship of gender and eating disorder symptoms to reported cravings for food: Construct validation of state and trait craving questionnaires in spanish. Appetite, 40, 47-54.

Christensen, L. (2007). Craving for sweet carbohydrate and fat-rich foods-possible triggers and impact on nutritional intake. Nutrition Bulletin, 32, 43-51.

Davis, C., \& Carter, J. C. (2014). If certain foods are addictive, how might this change the treatment of compulsive overeating and obesity? Current addiction reports (vol. 1 pp. 89-95).

Delahanty, L. M., Meigs, J. B., Hayden, D., Williamson, D. A., \& Nathan, D. M. (2002) Psychological and behavioral correlates of baseline BMI in the diabetes prevention program (DPP). Diabetes Care, 25, 1992-1998.

Edris, A. E. (2007). Pharmaceutical and therapeutic potentials of essential oils and their individual volatile constituents: A review. Phytotherapy Research, 21 308-323.

Frannie. (n.d.). Vanilla essential oil [product label]. (Wildomar, CA).

Haber, S. L., \& El-lbiary, S. Y. (2016). Peppermint oil for treatment of irritable bowe syndrome. American Journal of Health-System Pharmacy, 73, 22-31.

Hill, A. J. (2007). The psychology of food craving. Proceedings of the Nutrition Society, $66,277-285$

Jáuregui-Lobera, I., Bolaños-Rios, P., Valero, E., \& Prieto, I. R. (2012). Induction of food craving experience; the role of mental imagery, dietary restraint, mood and coping strategies. Nutricion Hospitalaria, 27, 1928-1935.

Kavanagh, D. J., Andrade, J., \& May, J. (2005). Imaginary relish and exquisite torture: The elaborated intrusion theory of desire. Psychological Review, 112, 446-467.

Kemps, E., \& Tiggemann, M. (2013). Olfactory stimulation curbs food cravings. Addictive Behaviors, 38, 1550-1554.

Kemps, E., \& Tiggemann, M. (2015). A role for mental imagery in the experience and reduction of food cravings. Frontiers in Psychiatry, 5, 1-4.

Kemps, E., Tiggemann, M., \& Bettany, S. (2012). Non-food odorants reduce chocolate cravings. Appetite, 58, 1087-1090.

Moffitt, R., Brinkworth, G., Noakes, M., \& Mohr, P. (2012). A comparison of cognitive restructuring and cognitive diffusion as strategies for resisting a craved food. Psychology \& Health, 27, 74-90.

Reed, J. A., Almeida, J., Wershing, B., \& Raudenbush, B. (2008). Effects of peppermint scent on appetite control and caloric intake. Appetite, 51, 393.

Rolls,, E. T., \& McCabe, C. (2007). Enhanced affective brain representation of chocolate in cravers vs. non-cravers. European Journal of Neuroscience, 26 1067-1076.

Rozin, P. (1982). "Taste-smell confusions" and the duality of the olfactory sense. Perception and Psychophysics, 31, 397-401.

Stafford, L. D., \& Whittle, A. (2015). Obese individuals have higher preference and sensitivity to odor of chocolate. Chemical Senses, 40, 279-284.

Tiggemann, M., \& Kemps, E. (2005). The phenomenology of food cravings: The role of mental imagery. Appetite, 45, 305-313.

Weingarten, H., \& Elston, D. (1991). Food cravings in a college population. Appetite, 17, 167-175.

Young Living Essential Oils, LC. (n.d.). Slique essence [product label], (Lehi, UT). 